\title{
Variable-state design parameters in the design of aero-structures made of intrinsically smart materials
}

\author{
A. Maheri \& A. T. Isikveren \\ Aerospace Vehicle Architecture \& Design Integration (AVADI) Research \\ Theme and Teaching Group, Department of Aerospace Engineering, \\ University of Bristol, $U K$
}

\begin{abstract}
The aerodynamic performance of aero-structures made of intrinsically smart materials, such as aeroelastic tailored wings and blades, cannot be obtained unless their material properties and structural characteristics are also known. The aerodynamic design of these structures is a naturally integrated design problem in which the set of design parameters comprises both aerodynamic and structural characteristics of the aero-structure. This paper references the inevitable problems arising when adopting an integrated design approach, suggesting an alternative approach for design of these structures. The concept of Variable State Design Parameters (VSDP) is introduced in this paper and it is explained how VSDP can be employed to convert the traditional integrated design process of intrinsically smart aero-structures, to a decoupled and hence computationally efficient design process. Through a design case study the practicality and efficiency of the new approach have been demonstrated.
\end{abstract}

Keywords: Variable State Design Parameter, smart structures, elastic coupling, aeroelastic tailoring, deign decoupling.

\section{Introduction}

The advent of smart materials has made the idea of shape-changing aerodynamic structures practical. Many research projects on using smart materials to control the shape of wings have been reported in the last decade, for instance see [1-4]. Simultaneously rotor blades made of smart materials have found many practical 
applications in helicopters [5,6] and potential applications in horizontal axis wind turbines [7-10]. Morphing aero-structures can be used either to enhance the performance of the system in a wider range of run-conditions or to preserve the performance against the effects of disturbances in the system. A morphing aero-structure, as a part of a performance controller, can be categorised as either active, when made of extrinsically smart materials with built in actuators, or passive, when made of intrinsically smart materials (elastic-coupled anisotropic composites). Passive aero-structures morph and change their topology in response to the variations of rotor run-condition or aircraft flight-condition. Analysis of these structures becomes complex when the aerodynamic loading is the source load for generating and controlling the induced deformation (i.e. bend-twist-coupled structures). In this case any variation in the aerodynamic loading changes the magnitude of the induced deformation; the induced deformation changes the flow kinematics and therefore affects the aerodynamic loading. Hence, in order to analyse the aerodynamic performance of these structures, an iterative coupled-aero-structure (CAS) analysis must be carried out [11]. A schematic description of a CAS analysis is shown in Figure 1.

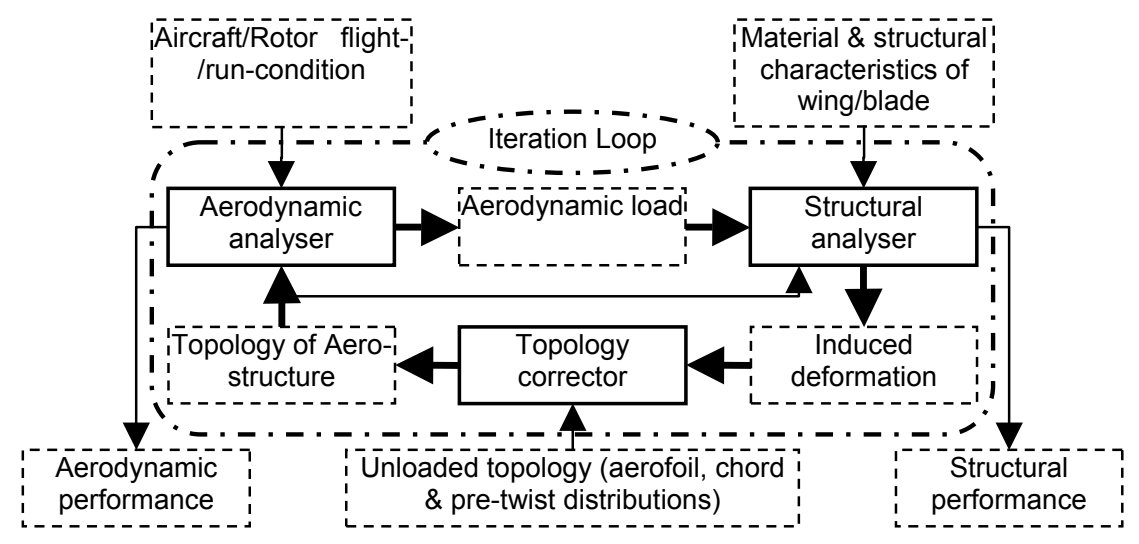

Figure 1: Iterative coupled-aero-structure analysis of passive smart wings/blades for a single flight-/run-condition.

\section{Integrated design}

Performing an integrated design has two main disadvantages, which make it inefficient in practice [12]. The first drawback is due to this fact that in an iterative CAS analysis a structural analyser must also be executed a number of times. Many investigations have been carried out towards closed form formulation of deformation of structures made of anisotropic composite materials. Finite element analysis (FEA), however, is still the only reliable means of precise determination of the induced deformation of these structures [13]. Utilising a FE-based code as a part of a design objective evaluator, makes the aerodynamic objective evaluation very time consuming [12]. Moreover, since 
in design of adaptive wings or blades the design objective is associated with the performance of the aircraft or rotor over a range of flight or run conditions, each objective evaluation is associated with a series of iterative CAS analyses at various flight-/run-conditions (states).

When evaluating the aerodynamic performance, in addition to the aerodynamic parameters (unloaded topology and size) many structural and material parameters are also involved (see Figure 2). This increases the number of parameters producing the design space and consequently the number of required evaluations grows exponentially. This is the second drawback of adapting an integrated design approach.

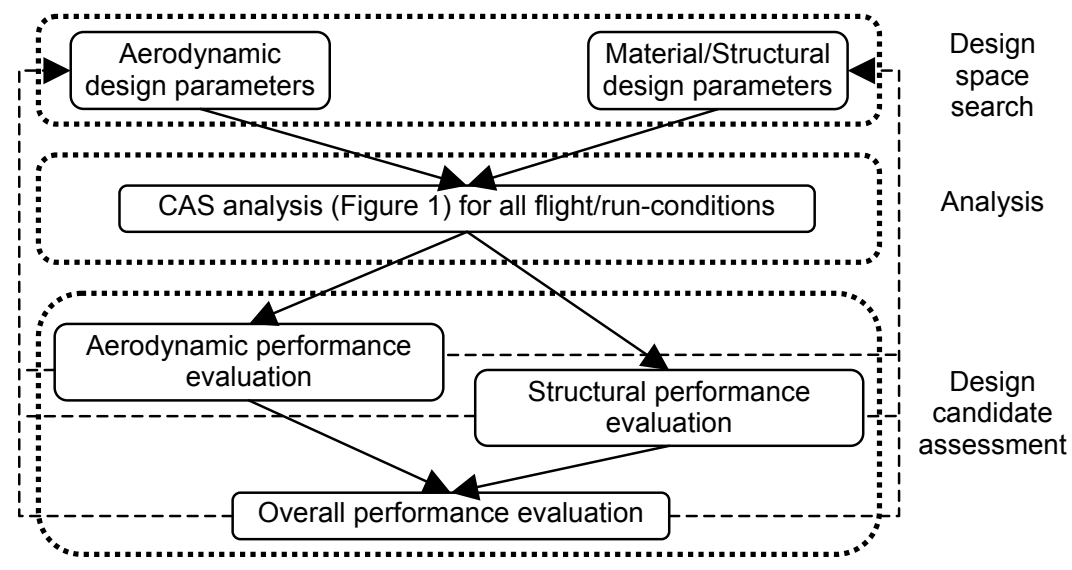

Figure 2: $\quad$ Integrated design.

\section{Induced deformation, a Variable State Design Parameter, (VSDP)}

It is the dependency of the induced deformation to both aerodynamic and structural characteristics of aero-structures that makes their analysis an iterative CAS process, hence their integrated design process very time consuming. The basis of the new approach into design of passive smart aero-structures is to introduce the induced deformation as an independent design parameter leading to breaking the coupling between aerodynamic and structural analyses of the aerostructure, and hence making the design process decoupled.

Adopting this approach has a twofold effect on reducing the computational time of design process. Firstly, an iterative CAS analysis is replaced by an iterative aerodynamic analysis, leading to significant reductions in analysis time. Secondly, since the aerodynamic and structural designs can be carried out separately in a sequential manner, the efficiency of the design space search improves significantly. 
Induced deformation depends on many parameters (unloaded topology, size, material and structural characteristics of the aero-structure) as well as the state. One may reintroduce the induced deformation $\varphi$ as follows

$$
\varphi=f\left(\text { state }, \varphi_{\text {ref }}\right)
$$

In which, the reference induced deformation $\varphi_{\text {ref }}$, is the induced deformation at a reference state and therefore is independent of the state variation. On the other hand, since for a given wing/blade (where the aero and material/structural parameters are fixed) the induced deformation is a function of the state only, one concludes that $\varphi_{\text {ref }}$ depends on the aero and material/structural parameters only. Deciding the aero and material/structural parameters by the designer makes the reference induced deformation $\varphi_{\text {ref }}$ a fixed value, whilst in contrast selecting $\varphi_{\text {ref }}$ as a design parameter, does not make aero and material/structural design parameters fixed. It only imposes one constraint into the design space.

Treating $\varphi_{r e f}$ as an ordinary design parameter, to be selected by the designer, the induced deformation $\varphi$ at various states can be determined accordingly if the function $f$ in Equation (1) is known.

In order to find the function $f$ in terms of state parameters one must perform a series of CAS analyses at various operating states for all possible aero and material/structural characteristics. However, a practical means of implementing VSDP into design is to define the function $f$ in terms of tangible intermediate parameters, for instance the dominant internal force producing the induced deformation. Maheri et al [13] showed that for a stall regulated wind turbine with bend-twist smart blades, the function $f$ has the following simple form:

$$
\varphi=\frac{M_{\text {root }}(\text { state })}{M_{\text {root }, \text { ref }}} \varphi_{\text {ref }}
$$

In which $\varphi$ stands for the induced twist, $M_{\text {root }}$ is the flap bending moment at the root of blade at a desired state and $M_{\text {root,ref }}$ is the flap bending moment at the root of blade at a reference state. The derivation of the above equation is based on two assumptions and one observation. The assumptions are (i) the edge-wise slope of the blade is negligible compared to the flap-wise slope and the twist of the blade and (ii) the internal torque due to the off-axis aerodynamic loading of the blade is negligible compared to the torque produced due to elastic coupling. Both of these assumptions are more or less accurate and valid for wings. Their observation was that the normalised flap bending moment in a blade, $M^{*}=M(x) / M_{\text {root }}$, is a weak function of wind turbine run-condition (wind speed, rotor speed and blade pitch angle). This observation is based on the analysis of the blade of a stall-regulated wind turbine, experiencing significantly different aerodynamic load distribution in its various operating scenarios. Since aircraft wings experience much smoother variations of aerodynamic loading at various 
flight-conditions (normal flight, gust and manoeuvre), the result of this observation can be applied to aircraft wings with higher levels of accuracy.

Bypassing determination of $\varphi$ as an explicit function of state parameters and representing $\varphi$ using intermediate parameters instead comes with the price of an iterative aerodynamic analysis as shown in Figure 3.

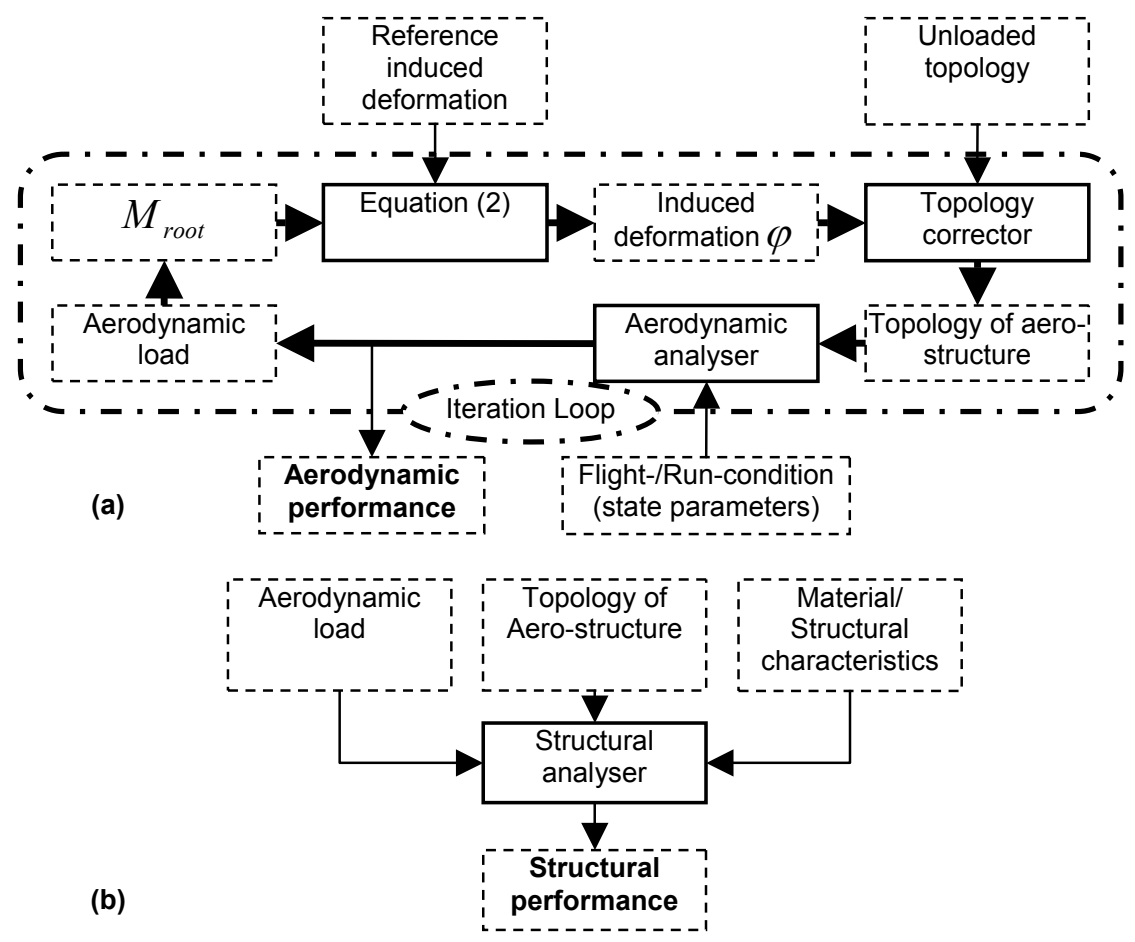

Figure 3: Decoupled (a) Aero and (b) Structure analysis with induced deformation as a VSDP.

\section{Design algorithm}

The design process of smart aero-structures using the induced deformation as a VSDP has been explained in Algorithm (1) and illustrated in Figure 4.

\section{Algorithm 1}

1. Select $\varphi_{\text {ref }}$ along other aerodynamic design parameters

2. Follow the algorithm of Figure 3(a) to find the converged values for the aerodynamic performance and aerodynamic loading of the aero-structure

3. If the aerodynamic performance is not satisfactory go back to Step 1; otherwise continue 
4. Select material/structural design parameters

5. Use the selected aerodynamic parameters as well as the aerodynamic forces obtained from the aerodynamic design (Steps 1 to 3 ) and perform structural analysis (Figure 3(b))

6. Evaluate the structural performance; if either the structural performance is not acceptable or the imposed constraint of having $\varphi=\varphi_{\text {ref, selected }}$ at the reference state is not satisfied go back to Step 4; otherwise continue

7. Evaluate the overall performance; if it is not satisfactory then go back to either Step 1 or Step 4; otherwise stop.

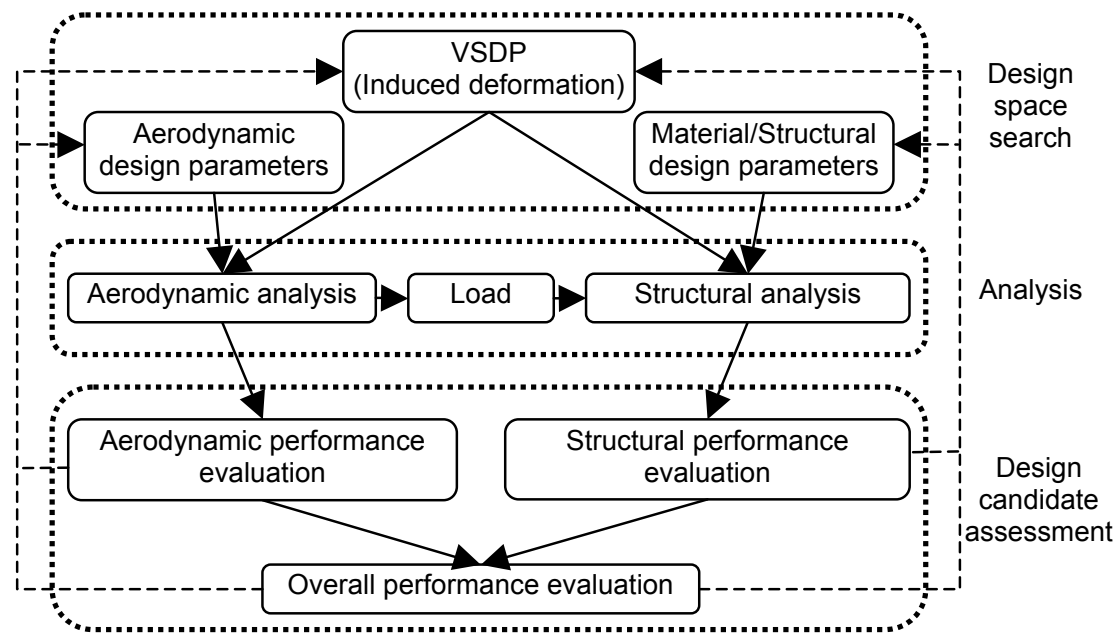

Figure 4: Decoupled design of passive smart aero-structures using Variable State Design Parameters.

In the explained design method, the aerodynamic and structural designs take place separately as for ordinary aerodynamic surfaces designs. The only difference between the proposed design methodology for passive smart aerostructures and traditional design methodology for ordinary aerodynamic surfaces is in (i) selection of $\varphi_{r e f}$ and (ii) the imposed constraint on structural design.

\subsection{On selection of $\varphi_{r e f}$ and the constraint imposed on structural design}

Span-wise trend and the amplitude of $\varphi_{\text {ref }}$ must be selected carefully avoiding situations in which the imposed structural constraint is impossible to be satisfied. Generally speaking, planning a particular span-wise trend for induced deformation is a difficult task that requires a great deal of effort and care on the selection of a proper span-wise material distribution and fibre angle variation.

Moreover, a wide range of material/structural parameters (e.g. ply angle, number of layers, thickness of the composite material and its fibre and matrix 
mechanical properties) affect the level of the produced elastic coupling and consequently the magnitude and distribution of the induced deformation in an aeroelastic tailored structure. Therefore the constraint of "having $\varphi=\varphi_{\text {ref,selected }}$ at the reference state" could be difficult to be fulfilled unless an appropriate search method for determination of material/structural design parameters is adopted. One may adopt an inverse method which employs a search tool operating based on artificial neural networks, whilst another approach is to reduce the structural design space in order to make the search for the set of material/structural design parameters easier. Here, reducing the structural design space refers to deciding some material/structural characteristics of the aerostructure prior to commencing the aerodynamic design.

\subsection{A design algorithm with reduced structural design space}

Maheri et al [14] showed that normalised induced deformation in a bend-twist shell member is a function of the dimensionless dominant internal force and effective stiffness. In other words, in a bend-twist wing/blade it is the normalised span-wise distributions of the effective stiffness and flap bending that dictate the distribution of the normalised induced twist $\varphi^{*}(x)=\varphi(x) / \varphi_{\max }$, not the actual values of those. This result coupled with the fact that the normalised flap bending distribution $M^{*}(x)$ is neither a function of material/structural characteristics nor state parameters, leads one to the conclusion that for a given material/structural configuration, once $\varphi^{*}(x)$ has been calculated it is valid for all states as well as all values of material/structural design parameters. The following algorithm is a modified version of Algorithm (1) using a reduced structural design space scheme.

\section{Algorithm 2}

1. Decide wing/blade material and structural configurations

2. For a set of arbitrary values of material/structural parameters run a CAS analysis to find the normalised induced deformation distribution $\varphi^{*}(x)=\varphi(x) / \varphi_{\max }$.

3. Replace $\varphi$ and $\varphi_{\text {ref }}$ with $\varphi_{\max }$ and $\varphi_{\text {max, ref }}$ respectively in Algorithm (1), treat $\varphi_{\max , \text { ref }}$ as a design parameter and follow that algorithm for the rest of the design process

\section{Variable State Design Parameters in practice - a case study}

This case study is aimed at to show the practicality and efficiency of the proposed design methodology by designing a bend-twist smart blade. The smart blade is intended to be replaced with the original blades in a constant-speed stallregulated WTA-27 wind turbine. The overall objective of the design and the objective of the aerodynamic design are both maximising the energy capture 
capabilities of the rotor. The aerodynamic design parameters are rotor radius $R$, pre-twist distribution $\beta_{0}$ and chord distribution $C$ as well as the induced twist at the tip of the blade at a reference state $\beta_{\text {max, ref }}$. A reference state of $V_{W, \text { ref }}=10 \mathrm{~m} / \mathrm{s}$ has been used for this study, where $V_{W, \text { ref }}$ stands for wind velocity. Two other state parameters, rotor speed and blade pitch angle, are constant for this wind turbine. It is assumed that the original aerofoil distribution has been used for the smart blade. The aerodynamic design is subject to the constraint of having the maximum rotor power limited to the original rated value. The adaptivity nature of smart blades allows the rotor to have a larger diameter at the same rotor speed as the original one [12]. Utilising longer span and twisting toward stall (as a necessity for enhancing the average power of the rotor) increase the blade loading. Therefore the structural design objective is selected as to maintain the stress level in the smart blade the same as of that in the original one. The ratio of $M / C^{3}$ has been used as an estimated measure for the stress level in the blade, where $M$ stands for the flap bending in the blade. For simplicity, weight and other structural behaviour of smart blade (i.e. tip deflection, inter-laminate stresses, natural frequency, etc) are not involved in this study.

It is assumed that the blade shell has been made of a constant thickness graphite epoxy composite and the bend-twist elastic coupling is generated due to mirror lay up of the entire upper and lower surfaces of the blade. That is the mechanical properties of the blade material (including the shell thickness) together with the fibre orientation are constant span-wise. In other words the material/structural configuration is constant span-wise and therefore the normalised induced twist distribution $\beta^{*}(x)$ is the same for all states as well as all material/structural design parameters. Ply angle and shell thickness are treated as two structural design parameters.

In order to find the wind turbine average power, a power curve must be generated. To generate a power curve the wind turbine must be simulated over its operational wind speed (here, a range of 5 to $25 \mathrm{~m} / \mathrm{s}$ with $1 \mathrm{~m} / \mathrm{s}$ increments).

WTAB [9], a simulation tool for wind turbines with passive smart blades has been employed to perform a CAS analysis of a blade having the original topology and size but made of graphite epoxy with a shell thickness of $10 \mathrm{~mm}$ and ply angle of 20 degrees which is running at a wind velocity of $10 \mathrm{~m} / \mathrm{s}$ with a rotor speed of $53.3 \mathrm{rpm}$. Figure 5 shows the normalised induced twist obtained from this analysis.

Using WTABD, a genetic algorithm-based design and optimisation code capable of performing Algorithms (1) and (2), the following results have been obtained:

- Rotor radius, $\mathrm{R}=14.3 \mathrm{~m}$ (3.8\% larger than the original radius);

- Pre-twist and chord distributions as shown in Figure 6

- A maximum induced twist (at the tip of the blade) of $\beta_{\text {max,ref }}=5.9^{\circ}$ (toward stall) at the reference state of $V_{W, r e f}=10 \mathrm{~m} / \mathrm{s}$. This identifies the level of elastic coupling required in the blade. 


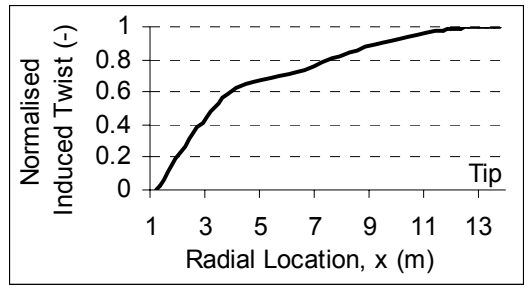

Figure 5: Normalised induced twist obtained from CAS analysis of an elastic-coupled blade.

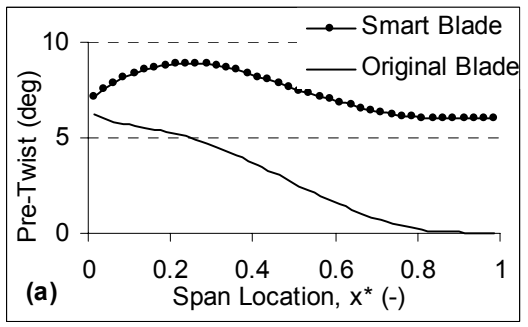

Figure 6: Designed smart blade distribution.

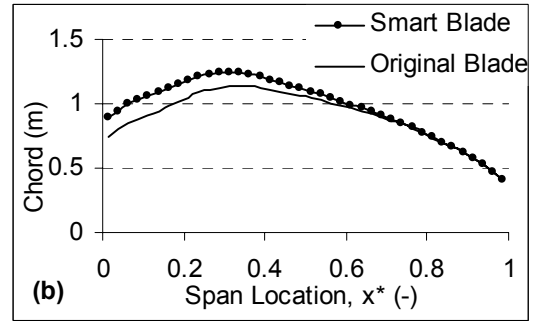

(a) pre-twist distribution (b) chord

Having fixed the blade topology from the aerodynamic design phase, WTAB can be employed again to find ply angle and shell thickness (in a trial and error basis). It is found that a ply angle of $\theta=22^{\circ}$ and a shell thickness of $t=6 \mathrm{~mm}$ generate the desired tip induced twist of $\beta_{T, \text { ref }}=5.9^{\circ}$ at the reference state.

The wind turbine that utilises these passive smart blades will produce an average power of $P_{a v}=48.4 \mathrm{KW}$ (at an average site speed of $V_{a v}=5.6 \mathrm{~m} / \mathrm{s}$ based on a Rayleigh probability distribution function), which is $16.9 \%$ greater than the original wind turbine.

If an integrated design approach was adopted in this study, assuming an average value of about 5 for the number of iterations in a CAS simulation [11], one had to run a structural analyser more than 100 times for each single objective evaluation.

\section{Conclusion}

Traditional methods of design of aerodynamic surfaces are not efficient when applied to design of intrinsically smart aero-structures. Introducing the induced deformation as a Variable State Design Parameter decouples the analysis of these structures therefore the aerodynamic and structural design of these structures can be carried out separately. The simplified design case studied in this paper shows that how adopting the proposed design methodology based on VSDP, enables one to design wind turbine smart blades efficiently without any structural analysis involved in the aerodynamic design phase. 


\section{References}

[1] Seigler, T.M., Neal, D.A., Bae, J. \& Inman, D.J., Modeling and Flight Control of Large-Scale Morphing Aircraft. Journal of Aircraft, 44(4), pp. 1077-1087, 2007.

[2] Sanders, B., Eastep, F.E. \& Forster, E., Aerodynamic and Aeroelastic Characteristics of Wings with Conformal Control Surfaces for Morphing Aircraft. Journal of Aircraft, 40(1), pp. 94-99, 2003.

[3] Campanile, L.F. \& Anders, S., Aerodynamic and Aeroelastic amplification in adaptive belt-rib airfoils. Journal of Aerospace Science and Technology, 9, pp. 55-63, 2005.

[4] Chopra, I., Review of State-of-Art of Smart Structures and Integrated Systems. AIAA Journal, 40(11), pp. 2145-2187, 2002.

[5] Ganguli, R. \& Chopra, I., Aeroelastic Tailoring of Composite Couplings and Blade Geometry of a Helicopter Rotor Using Optimization Methods. Journal of American Helicopter Society, 42(3), pp. 218-228, 1997.

[6] Singh, K., Sirohi, J. \& Chopra, I., An Improved Shape-Memory Alloy Actuator for Rotor Blade Tracking. Journal of Intelligent Material Systems and Structures, 14(12), pp. 767-786, 2003.

[7] Kooijman, H.J.T., Bending-Torsion Coupling of a Wind Turbine Rotor Blade. Netherlands Energy Research Foundation ECNI-96-060, December 1996.

[8] Eisler, G.R. \& Veers, P.S., Parameter Optimization Applied to Use of Adaptive Blade on a Variable-Speed Wind Turbine. Sandia National Laboratories SAND98-2668, December 1998.

[9] Maheri, A., Noroozi, S., Toomer, C., \& Vinney, J., WTAB, a Computer Program for Predicting the Performance of Horizontal Axis Wind Turbines with Adaptive Blades, Renewable Energy, 31(11), pp. 1673-1685, 2006.

[10] Maheri, A., Noroozi, S., Toomer, C., \& Vinney, J., A simple algorithm to modify an ordinary wind turbine blade to an adaptive one, Proc. of the European Wind Energy Conference, Athens, Greece, 27 February-2 March 2006.

[11] Maheri, A., Noroozi, S., Toomer, C., \& Vinney, J., Single step versus coupled aero-structure simulation of a wind turbine with bend-twist adaptive blades, Proc. of the European Wind Energy Conference, Athens, Greece, 27 February-2 March 2006.

[12] Maheri, A., Noroozi, S. \& Vinney, J., Application of combined analytical/FEA coupled-aero-structure simulation in design of wind turbine adaptive blades, Renewable Energy, 32(12), pp. 2011-2018, 2007.

[13] Maheri, A., Noroozi, S. \& Vinney, J., Combined Analytical/FEA-based Coupled-Aero-Structure Simulation of Wind Turbines with Bend-Twist Adaptive Blades, Renewable Energy, 32 (6), pp. 916-930, 2007.

[14] Maheri, A., Noroozi, S. \& Vinney, J., Decoupled design of wind turbine adaptive blades, Renewable Energy, 32(10), pp. 1753-1767, 2007. 